\title{
Análisis comparativo del uso de marcadores metadiscursivos en la evaluación formativa y sumativa online $^{*}$
}

\section{A comparative analysis of the use of metadiscourse markers in online formative and summative assessment}

\author{
Ma Luisa Carrió-Pastor \\ Universitat Politècnica de València \\ lcarrio@upv.es \\ ORCID ID: https://orcid.org/0000-0002-3040-5362
}

DOI: 10.17398/1988-8430.34.261

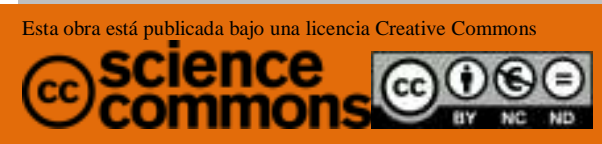

Fecha de recepción: $15 / 03 / 2021$

Fecha de aceptación: 26/04/2021

Carrió-Pastor, M. L. (2021). Análisis comparativo del uso de marcadores metadiscursivos en la evaluación formativa y sumativa online, Tejuelo, 34, 261-292.

Doi: https://doi.org/10.17398/1988-8430.34.261

\footnotetext{
* Este trabajo se ha realizado en el marco del Proyecto de Investigación IAMET (referencia FFI2016-77941-P).
} 
Resumen: Se propone en este artículo comparar la efectividad de la evaluación formativa y la evaluación sumativa al evaluar la comunicación en la realización de tareas online. Para ello, se diseñaron diferentes tipos de tareas online que los/as alumnos/as tenían que realizar en dos asignaturas, una del primer semestre y otra del segundo en el mismo curso académico. En el primer semestre se aplicó la evaluación sumativa al grupo de control y en el segundo semestre la evaluación formativa al grupo experimental. Se realizó este experimento con alumnos de posgrado cuya lengua materna era el chino y tenían que realizar las tareas en español. Con el fin de identificar y analizar los resultados del experimento se tuvieron en cuenta varios parámetros como el uso de marcadores metadiscursivos, la interacción con el profesor y la comunicación efectiva. Los/as alumnos/as realizaron las tareas de forma progresiva y se compararon los resultados en el grupo de control y el experimental. Se comentaron los resultados con el fin de identificar si se observaban diferencias en el uso de marcadores metadiscursivos y se extrajeron las conclusiones del estudio.

Palabras clave: evaluación sumativa; evaluación formativa; marcadores; metadiscurso; hablantes no nativos.
Abstract: In this paper, my main aim is to compare the effectiveness of formative assessment and summative assessment of communicative skills in online tasks. Different types of online tasks were designed to be done by students in two subjects in the first and second semester of an academic year. Summative assessment was carried out in the subject of the first semester to the control group. In the second semester, formative assessment was performed in another subject to the experimental group. This experiment was performed to graduate students whose mother tongue was Chinese and had to write the tasks in Spanish. In order to identify and analyse the results of the experiment, several aspects were taken into account, such as the use of metadiscursive markers, interaction with the teacher, and effective communication. The students did the tasks progressively and the results were compared in the control and experimental groups. The results were commented in order to identify if differences were observed in the use of metadiscursive markers and the conclusions of the study were drawn.

Keywords: summative assessment; formative assessment; markers; metadiscourse; non native speakers. 


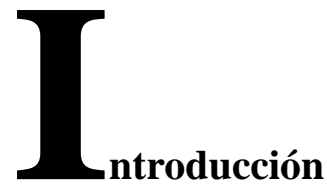

Este estudio se ha realizado en un momento en que corresponde cuestionarnos la forma en que enseñamos, lo cual nos lleva a afrontar nuevos retos y horizontes en la investigación en didáctica de la lengua. La pandemia ocasionada por el COVID-19 ha hecho que los docentes se plateen nuevos experimentos y formas de enseñar. En este trabajo se estudia el uso de los marcadores metadiscursivos en la contestación a tareas por parte de alumnos cuya lengua materna no es el español; es decir, se evalúa no solo su conocimiento, si no también su capacidad persuasiva y comunicativa. Para ello se realizaron tareas de evaluación formativa y de evaluación sumativa con el fin de comparar el uso de los marcadores discursivos en sus respuestas a las diversas tareas. Ello nos ayudó a identificar qué forma de evaluación podría ser más efectiva y si se mejoraba su forma de comunicación en la lengua meta tras el proceso de la evaluación sumativa y el de la formativa.

En este estudio se tiene en cuenta la importancia de dominar el uso de los marcadores metadiscursivos (Hyland, 2005) para lograr una 
comunicación efectiva. Es por ello que, partiendo de una evaluación sumativa, me percaté de la dificultad de los sinohablantes para poder comunicarse de forma correcta en una titulación cuya lengua vehicular es el español y diseñé actividades que permitían la evaluación formativa para poder comprobar si se mejoraba la producción de actividades por parte de los/as alumnos/as cuya lengua materna es el chino.

En este artículo se muestra el experimento que se llevó a cabo con el fin de comparar dos tipos de evaluación en una situación en la que no se podía tener interacción con los/as alumnos/as por la pandemia del COVID-19 y se debía de evaluar en una plataforma online con fines educativos. Después de esta experiencia, en este artículo se propone comparar la efectividad de la evaluación formativa y la evaluación sumativa en la realización de tareas en español online por hablantes no nativos.

Los objetivos de este análisis son, por un lado, comparar dos tipos de evaluación para identificar su eficacia en lo que respecta al uso de la lengua española por parte de hablantes no nativos. Por otro, se pretende determinar si el uso de los marcadores metadiscursivos y, con ello, la capacidad expositiva y persuasiva de los/as alumnos/as, mejora con la evaluación formativa. El tercer y último objetivo es analizar la interacción entre profesores y alumnos en las actividades online, así como si la comunicación fue efectiva, identificando patrones. Como consecuencia, las preguntas de investigación que se plantean son las siguientes:

a) ¿Es efectiva la evaluación formativa para mejorar la comunicación en una lengua extranjera?

b) ¿Qué tipo de interacción entre profesor y alumno en la evaluación formativa es más efectiva y produce más actos comunicativos?

Para lograr los objetivos trazados y contestar a las preguntas de investigación, este artículo se ha divido en varias secciones. En primer lugar, se describen los marcadores metadiscursivos y su papel en la comunicación efectiva. A continuación, se identifican las características 
de la evaluación formativa y la evaluación sumativa y cómo se aplican en la enseñanza de lenguas. Una vez finalizada la revisión de la fundamentación teórica, se describe el material recopilado y el método utilizado. En la siguiente sección se detallan y comentan los resultados del análisis, para finalizar con las conclusiones que responden a los objetivos y las preguntas de investigación.

\section{Los marcadores metadiscursivos}

La conceptualización pragmática del uso de las lenguas es un aspecto ampliamente estudiado. Específicamente, Crystal (1997, p. 301) la define como "[...] the study of language from the point of view of users, specially of the choices they make, the constraints they encounter in using language in social interaction and the effects their use of language has on other participants in the act of communication". Con el fin de identificar la competencia pragmática de los hablantes de una lengua, se suele distinguir entre la sociopragmática, es decir, conocer las normas y convenciones sociales del uso de una lengua, y la pragmalingüística, que implica saber utilizar la lengua de forma adecuada para poder realizar correctamente diferentes actos de habla de forma convencional (Roever, 2014).

Esta fundamentación teórica sobre la pragmática es la que subyace en los estudios interlingüísticos e intralingüísticos (Connor, 1996, Carrió-Pastor, 2021), pilares de la evaluación del uso de la pragmática en una lengua extranjera. Uno de los aspectos que ha despertado más interés en los análisis pragmáticos es el uso del metadiscurso, tanto estudiando cómo se utiliza en la lengua materna (Thompson, 2001, Hyland, 2005, Mur-Dueñas, 2011, Salas Valdebenito, 2015, Carrió-Pastor, 2016a, 2019) como en la lengua extranjera (Connor, 1996, Carrió-Pastor, 2016b, 2021). Estos estudios han identificado marcadores discursivos que se utilizan en el lenguaje académico para guiar al lector o persuadirlo sobre sus ideas y se basan en los estudios de Halliday (1998) sobre la interpretación social de la lengua. 
Existen varias definiciones del metadiscurso, entre ellas destacamos las apuntadas por Connor (1996, p. 94) "[...] el metadiscurso le permite al escritor mostrar al lector cómo las partes del texto se relacionan entre sí y expresar su propia evaluación del contenido y su actitud", o por Hyland (1998):

\begin{abstract}
[...] metadiscourse is not an independent stylistic device which authors can vary at will. It is integral to the contexts in which it occurs and is intimately linked to the norms and expectations of particular cultural and professional communities. Writing is a culturally situated social activity and effective metadiscourse use is critically dependent on a rhetorical context and the writer's observation of appropriate interpersonal and intertextual relationships. To understand the pragmatics of metadiscourse, then, it must be located in the settings which determine its use and give it meaning (p. 438).
\end{abstract}

Por lo tanto, los aspectos a destacar en el uso adecuado del metadiscurso son el contexto, el género y la adecuación linguiística a la situación comunicativa para, con ello, lograr la interacción con el oyente. Muchos de los estudios sobre el metadiscurso identifican dos categorías con dos funciones distintas (Thompson, 2001, Hyland, 2005, Mur Dueñas, 2011). Por un lado, la categoría textual, que es la que aglutina los marcadores discursivos que sirven de apoyo para estructurar y darle forma al discurso, tales como los conectores, ejemplificadores, secuenciadores, etc., cuyo fin es conferir coherencia y cohesión a la lengua. Por otro, en la categoría interpersonal se incluyen los marcadores discursivos que confieren un significado determinado al texto y que ayudan al emisor a persuadir al receptor y a crear una imagen de sí mismo, como, por ejemplo, los atenuadores, los enfatizadores, etc.

El uso de los marcadores metadiscursivos en español ha sido de interés para diversos investigadores como Briz (2007), Albelda y Cesteros (2011), Salas Valdebenito (2015), Moya y Carrió-Pastor (2018) y Flores (2020). Estos investigadores han identificado los diferentes marcadores metadiscursivos en diversos contextos para proporcionarnos sus distintas funciones con el fin de conocer en profundidad las implicaciones pragmáticas de la comunicación. En este artículo se han tenido en cuenta estos estudios para identificar y conocer 
la función de los marcadores metadiscursivos y, con ello, ayudar a hablantes no nativos a mejorar la comunicación en español.

Las dos categorías apuntadas anteriormente se dividen en varias subcategorías que identifican la función de los marcadores y específicamente como se han de utilizar en el discurso. Respecto a la categoría textual, podemos identificar las siguientes subcategorías, que se pueden ver en la tabla 1:

\section{Tabla 1}

Subcategorías de los marcadores textuales

\begin{tabular}{|c|c|c|}
\hline Metadiscurso textual & Definición & Tipos \\
\hline Marcadores lógicos & $\begin{array}{l}\text { Relacionan unos elementos con otros, } \\
\text { enlazando los enunciados y guiando el } \\
\text { procesamiento de la información }\end{array}$ & $\begin{array}{c}\text { Aditivos } \\
\text { Adversativos } \\
\text { Consecutivos }\end{array}$ \\
\hline Códigos de glosa & $\begin{array}{l}\text { Explican el significado de la } \\
\text { proposición }\end{array}$ & $\begin{array}{l}\text { Reformulación } \\
\text { Ejemplificación }\end{array}$ \\
\hline Secuenciadores & $\begin{array}{l}\text { Secuenciación del discurso, marcan el } \\
\text { ritmo de la argumentación }\end{array}$ & - \\
\hline Topicalizadores & $\begin{array}{l}\text { Indican el tema, focalizando la } \\
\text { atención sobre el punto principal }\end{array}$ & - \\
\hline $\begin{array}{l}\text { Marcadores del } \\
\text { discurso }\end{array}$ & $\begin{array}{l}\text { Guían al receptor no desde el punto de } \\
\text { vista de la coherencia de los párrafos, } \\
\text { sino de todo el texto }\end{array}$ & - \\
\hline Enunciadores & Indican la intención del hablante & - \\
\hline Endofóricos & $\begin{array}{l}\text { Apuntan o señalan, en forma anafórica } \\
\text { o catafórica, a otras partes del discurso }\end{array}$ & $\begin{array}{l}\text { Anafóricos } \\
\text { Catafóricos }\end{array}$ \\
\hline Evidenciales & $\begin{array}{l}\text { Indican que existen evidencias de lo } \\
\text { indicado en una proposición }\end{array}$ & $\begin{array}{l}\text { Personales } \\
\text { Impersonales }\end{array}$ \\
\hline
\end{tabular}

Fuente: Carrió-Pastor (2020) 
Respecto a los marcadores lógicos, las diferencias entre ellos se basan en que los marcadores aditivos añaden información, algunos ejemplos son: además, por otro lado, etc., Los marcadores adversativos o de contraste contraponen un sentido a otro, algunos ejemplos son: sin embargo, pero, etc. y los marcadores continuativos o consecutivos tienen un valor de cohesión interna, ejemplos son: pues, por ese motivo, etc.

Los códigos de glosa se dividen en marcadores de reformulación, que reformulan una idea como son: es decir, en concreto, esto es, etc. y en marcadores de ejemplificación, los cuales muestran ejemplos al receptor, algunos ejemplos son: como, tales como, etc.

Algunos ejemplos de los secuenciadores son primero, segundo, a continuación, finalmente, etc. Ejemplos de los topicalizadores son: en cuanto $a$, respecto $a$, etc. Los marcadores del discurso son elementos como hasta ahora, en resumen, etc. Los enunciadores más comunes son vamos a, seguimos, etc. Respecto a los marcadores endofóricos, los anafóricos se refieren a partes anteriores del discurso, algunos ejemplos son: por ejemplo, anterior, previo, al principio, antes, etc. y los catafóricos a partes del discurso posteriores, que se realiza con los marcadores: por ejemplo, siguiente, seguidamente, a continuación, etc.

En lo que concierne a los marcadores evidenciales, se ha adoptado la concepción de Hyland (2005), aunque existan otros estudios que utilizan este término para referirse a diversas interpretaciones funcionales. Respecto a los personales, indican la evidencia en modo de persona, con marcadores como: como señala $X$. Los impersonales se usan para referirse a una tabla, un gráfico o figura como evidencia, por ejemplo, con: como se indica en la tabla, etc.

La segunda categoría de marcadores metadiscursivos, la interpersonal, se divide en las subcategorías que se ven en la tabla 2: 


\section{Tabla 2}

Subcategorías de los marcadores interpersonales

\begin{tabular}{|c|c|c|}
\hline $\begin{array}{l}\text { Metadiscurso } \\
\text { interpersonal }\end{array}$ & Definición & Tipos y ejemplos \\
\hline $\begin{array}{l}\text { Atenuadores o } \\
\text { mitigadores }\end{array}$ & $\begin{array}{l}\text { Mitigan la opinión o aseveración del } \\
\text { emisor, con el fin, muchas veces de } \\
\text { persuadir al receptor }\end{array}$ & $\begin{array}{l}\text { poder, considerar, } \\
\text { intentar, apuntar, } \\
\text { bastante, casi, etc. }\end{array}$ \\
\hline $\begin{array}{l}\text { Intensificadores o } \\
\text { enfatizadores }\end{array}$ & $\begin{array}{c}\text { Enfatizar la opinión o la proposición } \\
\text { que se realiza }\end{array}$ & $\begin{array}{l}\text { mostrar, determinar, } \\
\text { constatar, cierto, } \\
\text { evidente, etc. }\end{array}$ \\
\hline $\begin{array}{l}\text { Marcadores } \\
\text { actitudinales }\end{array}$ & $\begin{array}{l}\text { Expresan la evaluación o apreciación } \\
\text { que el hablante hace de los hechos } \\
\text { explicados en una proposición }\end{array}$ & $\begin{array}{l}\text { principal, importante, } \\
\text { relevante, gran, } \\
\text { necesario, bueno, } \\
\text { adecuado, etc. }\end{array}$ \\
\hline $\begin{array}{l}\text { Marcadores } \\
\text { relacionales }\end{array}$ & $\begin{array}{l}\text { Involucran a los receptores en el } \\
\text { discurso y se dirigen a ellos, hacen que } \\
\text { el receptor se sienta parte del mensaje y } \\
\text { que se involucre en la comunicación }\end{array}$ & $\begin{array}{c}\text { Directivos personales: } \\
\text { comencemos, miremos, } \\
\text { vosotros, etc. } \\
\text { Directivos: deber, } \\
\text { haber que, etc. }\end{array}$ \\
\hline Automenciones & $\begin{array}{l}\text { Se refieren a los narradores de la } \\
\text { comunicación }\end{array}$ & $\begin{array}{l}\text { Referencias personales: } \\
\text { comenzamos, nuestro, } \\
\text { etc. } \\
\text { Autocitas }\end{array}$ \\
\hline
\end{tabular}

Fuente: Carrió-Pastor, 2020

Los marcadores metadiscursivos forman parte intrínseca de la comunicación, ya que los hablantes utilizamos estos recursos retóricos que nos permiten organizar el pensamiento $\mathrm{y}$, de la misma forma, convencer o persuadir a los receptores del mensaje de nuestras intenciones. Por ello, utilizar los marcadores metadiscursivos de forma correcta es esencial para la comunicación e interacción. Han de ser utilizados de forma efectiva por los hablantes no nativos de una lengua, puesto que pueden tener una forma y/o función distinta en cada lengua. Por ello, es importante evaluar de forma eficaz el uso de una lengua 
extranjera para poder identificar los usos pragmáticos incorrectos. En este estudio se ha tenido en cuenta esta taxonomía de los marcadores metadiscursivos basada, sobre todo, en la tradición de estudios sobre la lengua inglesa, la cual hemos adaptado al análisis del español, valorando la función comunicativa de los marcadores y la intención del autor con respecto al lector.

\section{La evaluación sumativa y la evaluación formativa}

Otros dos aspectos que se han tenido en cuenta en este estudio son la evaluación sumativa y la evaluación formativa. La forma en que se lleva a cabo la evaluación de un proceso de aprendizaje es esencial para poder cumplir los objetivos que se diseñan en el programa. En las instituciones de educación superior se les suele solicitar a los profesores que evalúen el rendimiento de los/as alumnos/as de forma numérica, sin tener en cuenta el proceso previo que se ha llevado a cabo antes de la evaluación final. En este estudio comparamos el proceso y los resultados de la evaluación sumativa y la evaluación formativa; para ello, primero vamos a describir las diferencias entre los dos tipos de evaluaciones y las principales investigaciones llevadas a cabo en la evaluación sumativa y la evaluación formativa, teniendo en cuenta también los estudios llevados a cabo sobre la evaluación formativa online.

En lo que respecta a la evaluación sumativa, se basa en identificar una nota media en la que se incorporan los resultados que se obtienen al final de un proceso de enseñanza-aprendizaje. Se tienen en cuenta los diferentes actos de evaluación realizados para medir los conocimientos de los/as alumnos/as y su función es determinar si se han logrado los objetivos del programa de una asignatura. Así mismo, aporta una nota exacta de cada alumno que nos permite identificar un valor con el que podemos comparar el aprendizaje de un alumno con el del resto.

Mediante la evaluación sumativa se valora el rendimiento global del alumno y del proyecto educativo y se tiene en cuenta un punto de 
referencia para identificar el rendimiento, como, por ejemplo, la nota mínima es 0 o D y la máxima es 10 o A. Con ello se sabe si el alumno ha adquirido los conocimientos, destrezas y competencias previstos por el profesor y puede continuar con posteriores aprendizajes o, si, por el contrario, no los ha adquirido y ha de continuar con ese aprendizaje, ya que se ha de asegurar que los/as alumnos/as alcanzan las metas educativas propuestas. La evaluación sumativa se mide con puntos o calificaciones y se realizan a través de exámenes escritos o pruebas orales.

Por otro lado, la evaluación formativa tiene como objetivo acompañar al alumno en el proceso de aprendizaje y proporcionarle retroalimentación de las tareas realizadas. Granberg et al. (2021) nos indican los siguiente:

When putting formative assessment into practice, the teacher gathers evidence of the students' learning, and based on the identified learning needs, adapts instruction or feedback to meet these needs. The students may also be the agents of these formative assessment practices through peer assessment and self-assessment, including feedback to their peers or themselves. The teacher's role in accomplishing a formative assessment practice in which the students are active agents in the core formative assessment processes is to help students become motivated and proficient in carrying out these processes. In addition, in order for formative assessment practices to be most effective, the teacher may work together with the students to achieve a mutual understanding of the learning goals and establish criteria for attainment of these goals (p. 1).

Con ello, los/as alumnos/as van aprendiendo a la vez que son evaluados, avanzando en el aprendizaje a través de la evaluación, que deja de ser un acto realizado por el profesor y se convierte en un proceso evaluador en el que están involucrados los/as alumnos/as y los profesores. Con este tipo de evaluación se identifican las áreas o aspectos a mejorar basándose en una forma de aprender constructiva. Las pruebas más comunes son ejercicios en los que los/as alumnos/as realizan resúmenes, explicaciones, mapas conceptuales, etc. Varios son los investigadores que apoyan este tipo de evaluación, como Black (2015) y Sutton (2010). Este último, en concreto identifica las siguientes acciones a llevar a cabo (Sutton, 2010, p. 355): 
1. Planificación: el propósito de la planificación de los profesores ha de ser la enseñanza, no el cumplir objetivos.

2. Cuestionamiento: uno de los propósitos de la evaluación formativa es animar a los/as alumnos/as a pensar y cuestionar lo que aprenden.

3. Las notas y la retroalimentación: Los profesores han de establecer criterios claros que sean comprendidos por los aprendices.

Si comparamos los dos tipos de evaluación, algunos investigadores (Gulikers et al., 2013) proponen que haya una alienación entre la evaluación sumativa y formativa, con lo cual los profesores tendrían que adaptarse a las nuevas formas de evaluación. Indican que "[...] teachers never thought of formative assessment as being inevitable connected to the content or form of the summative assessment" (Gulikers et al., 2013, p. 122). Por lo tanto, recomiendan que los profesores planteen la incorporación de la evaluación formativa como parte del sistema educativo en el que incorporan aspectos innovadores que aplican de igual forma a la evaluación sumativa que es la que se realiza mayoritariamente en la actualidad. En la Figura 1 se muestra una propuesta de alienación de la evaluación formativa, comparando ideas preconcebidas sobre la evaluación formativa con lo que realmente ha de implantarse:

\section{Figura 1}

Propuesta de evaluación formativa centrada conceptualmente

\begin{tabular}{|c|c|}
\hline Current coeceptions &  \\
\hline 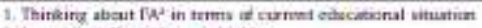 & 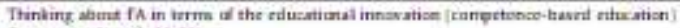 \\
\hline 2. Irpuitansapline erientad thirking & Dutcome-criented thinkins \\
\hline 3. Thitkirs in prooks a brawledgr per courat & Thating in student activities ahiwims perfumanze of atatrones \\
\hline 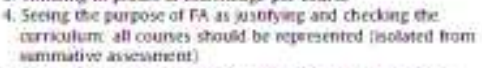 & 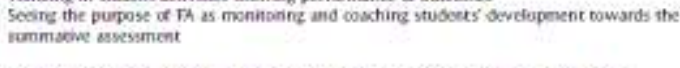 \\
\hline 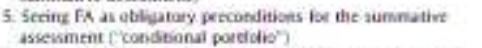 & 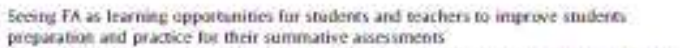 \\
\hline 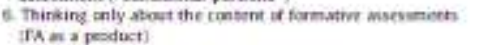 & 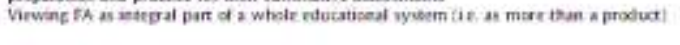 \\
\hline 7. Thinaing abcut $\mathrm{FA}$ as the end of imstruction. & Thinking about Fk as part of imatrcation \\
\hline 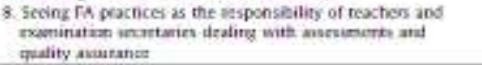 & 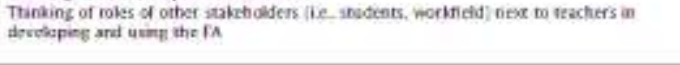 \\
\hline
\end{tabular}

Fuente: Gulikers et al., 2013, p. 121 
En la figura se destaca que la evaluación formativa es parte de la innovación educativa y que su fin último es la educación integral del alumno, siendo parte de la instrucción. Gulikers et al. (2013) así como Yin y Buck (2019) enfatizan que se ha de considerar la combinación de la evaluación formativa y sumativa desde un punto de vista colaborativo, en el que la planificación, las acciones, la observación y la reflexión son esenciales. La colaboración se puede realizar de forma conjunta con otros profesores o entre los/as alumnos/as y los profesores e incluso los padres (Pine, 2009), con el fin de armonizar el uso de la evaluación sumativa y la formativa.

La tecnología también juega un papel importante en la combinación de los dos tipos de evaluaciones sobre todo actualmente, ya que nos vemos forzados a evaluar con el uso de plataformas online o herramientas tecnológicas. Mohamadi (2018) compara la efectividad de la evaluación sumativa y formativa de la escritura llevada a cabo de forma online, indicando que la evaluación formativa realizada en grupos de alumnos mejora la forma en que interaccionan y cómo desarrollan la producción final, ya que se ofrece retroalimentación no solo de los profesores, sino también de los compañeros. Este autor destaca que la interacción entre los/as alumnos/as facilita que el profesor observe sus progresos, así como le ayuda a diseñar el siguiente paso en el aprendizaje.

Así pues, nos encontramos actualmente con que en la mayoría de las universidades españolas se suele utilizar un modelo de evaluación sumativa, puesto que tradicionalmente es la que nos ha indicado una nota final de cada alumno que nos facilita saber en qué grado se ha esforzado, lo que ha comprendido en clase y de qué forma lo puede transmitir. Sin embargo, los investigadores sobre evaluación son conscientes de la importancia de introducir nuevas formas de valorar el aprendizaje de los/as alumnos/as y, por lo tanto, la aplicación de la evaluación formativa ha generado interés. Entre los diversos estudios sobre este tema destacamos los de Black (2010), Tillema (2010), Marshall (2010); sobre todo ha habido una gran muestra de interés en los últimos diez años, como lo demuestran Havnes et al. (2012), Panadero y Johnson (2013), Hamodi et al. (2015), Talanquer (2015), 
Meusen-Beekman et al. (2016), Pla-Campas et al. (2016), De la Hoz Ibacache (2016), Andersson y Palm (2017) y Hansen y Ringdal (2018). Estos estudios destacan los aspectos positivos de la evaluación formativa describiendo diversos experimentos llevados a cabo en distintos niveles de enseñanza y prestando especial atención a cómo adaptan y aplican los profesores esta nueva forma de evaluación.

Más recientemente, investigadores como Van der Kleij (2019), y Yang (2019), Hansen (2020), Schildkamp et al. (2020) y Granberg et al. (2021) inciden en los efectos de la evaluación formativa en los profesores y los/as alumnos/as, destacando hallazgos sobre la autoregulación del aprendizaje. Como indican Xiao y Yang (2019, pp. 3941): "Self-regulated learners are able to define their goals of learning and pursue actions of knowledge on their own initiative [...] formative assessment and feedback have the potential to help students become self-regulated learners". Así pues, la auto-regularización sería uno de los aspectos fundamentales a tener en cuenta como fruto de la evaluación formativa.

Para finalizar con los estudios que se han tenido en cuenta en este artículo, el uso de la tecnología en la evaluación formativa ha sido de interés para diversos investigadores. Concretamente, el uso de plataformas online que se utilizan para la evaluación formativa ha permitido que se realicen varios experimentos. Varias investigaciones como las de Pellegrino (2010), Costa et al. (2010), Pachler et al. (2010), Gikandi et al. (2011), Wilson et al. (2017) y Fernando $(2018,2020)$ nos detallan sus ventajas e inconvenientes. Estos autores han destacado principalmente que la tecnología favorece la evaluación formativa y el aprendizaje. Sobre todo, destacan que existe una interacción más inmediata entre el alumno y el profesor, así como la evaluación entre pares, aunque algunos reconocen que la evaluación formativa automatizada no es tan fiable $\mathrm{y}$, en ocasiones, desmotiva a los/as alumnos/as si la interacción en el proceso de la evaluación no es efectiva. Sobre todo, se destaca la importancia del uso de la tecnología en la evaluación formativa en las universidades (Gikandi et al., 2011), ya que los/as alumnos/as son capaces de autorregular su aprendizaje de forma más eficaz y ello conlleva que la evaluación formativa sea más 
efectiva que la sumativa, ya que les motiva más y les fuerza a seguir un ritmo de aprendizaje constante y no están pendientes de un examen final.

\section{Material y método}

A continuación, en esta sección se describen el material, participantes y el método utilizado para el experimento que se ha llevado a cabo con el fin de comparar la evaluación sumativa y formativa llevada a cabo de forma online en un ámbito universitario.

\section{1. Material y participantes}

Para este estudio se seleccionaron y analizaron dos corpus. Por un lado, un corpus estaba compuesto por tareas de la evaluación sumativa de una asignatura realizadas por sinohablantes durante el primer cuatrimestre del curso 2020-21 en un título de posgrado de la Universitat Politècnica de València. Este corpus se recopiló para estudiar lo que denominamos el grupo de control.

El segundo corpus incluía las actividades que se realizaron para la evaluación formativa de los mismos alumnos en otra asignatura del segundo cuatrimestre de ese curso y título de posgrado. Este corpus se recopiló para analizar el que denominamos grupo experimental.

Así pues, todo el material para el estudio comparativo se recopiló durante el primer y segundo cuatrimestre del curso en dos asignaturas diferentes. Los participantes que colaboraron en el estudio fueron, en el primer cuatrimestre, 24 alumnos/as chinos/as que se matricularon en el Máster en Lenguas y Tecnología de la Universitat Politècnica de València, veinte eran mujeres y cuatro hombres. Su edad estaba comprendida entre 24 y 30 años, hablaban español a nivel de usuario avanzado y realizaron todos los exámenes en lengua española.

En el segundo cuatrimestre, el grupo que fue estudiado estaba compuesto de un total de dieciocho alumnas chinas, que eran parte de 
las matriculadas en la asignatura del primer cuatrimestre. Todas las clases eran impartidas en español y las actividades y la retroalimentación se realizaban en español. Su edad estaba comprendida entre 24 y 28 años.

Los alumnos y las alumnas se inscriben en el Máster para adquirir nociones de lingüística, pero evaluar sus tareas y exámenes, para llevar a cabo una correcta interpretación y evaluación, lleva implícito la corrección de contenido y también de la lengua española. Todos/as los/las alumnos/as sinohablantes del Máster son admitidos con una certificación de nivel $\mathrm{C} 1$ en la lengua española. Cada curso académico se reciben muchas solicitudes por parte de alumnos/as chinos/as, ya que desean ser docentes o dedicarse a la traducción en China y finalizar un máster en España es un mérito notable para su currículum. Son conscientes de las dificultades que la comunicación en otra lengua implica, pero están muy motivados, ya que su intención es perfeccionar su español.

$\mathrm{Su}$ actitud en todo el proceso de la investigación fue muy positiva y estuvo motivada porque deseaban comprender las distintas formas de evaluación que llevábamos a cabo, es decir, aprendían a la vez que participaban en el experimento.

\section{2. Método}

Para recopilar los dos corpus, el del grupo de control y el del experimental, se aplicaron dos tipos de evaluación a estos alumnos con el fin de comparar sus avances en la adquisición de capacidades comunicativas. Para ello, al inicio del curso se diseñaron las actividades del grupo de control y del grupo experimental. Las actividades de la evaluación sumativa de la asignatura del primer cuatrimestre eran las que se estaban realizando tradicionalmente en las dos asignaturas y se revisaron para comprobar si se adecuaban al experimento. La evaluación en las dos asignaturas se realizó online. La evaluación de las actividades escritas se hizo a través de la plataforma PoliformaT, que es la versión 11.3 de Sakai, y la de las actividades orales a través de Microsoft Teams. PoliformaT está compuesta de varias secciones o 
unidades en las que se incluyen las tareas o actividades a realizar por los/las alumnos/as. Véase en la Figura 2 la interfaz de una de las asignaturas:

\section{Figura 2.}

\section{Interfaz de PoliformaT}

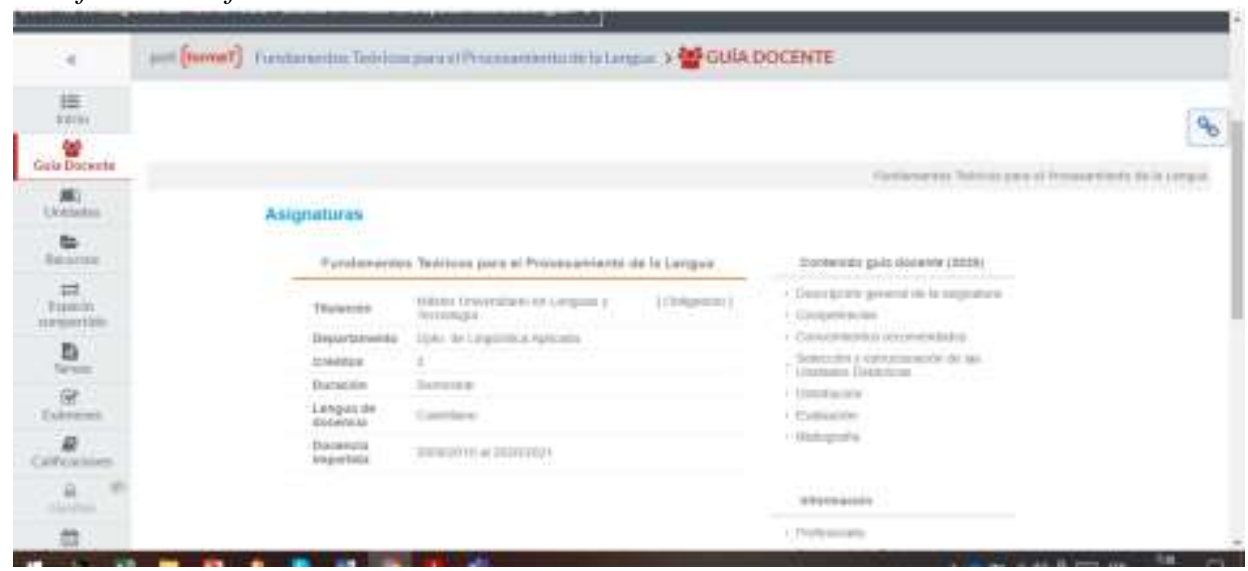

Fuente: PoliformaT

Como se puede observar en la Figura 2, las unidades, la información sobre la asignatura, las tareas y exámenes están disponibles en el menú de la izquierda. Esta herramienta incluye un chat y un foro para realizar actividades grupales, así como una sección de video apuntes en la que los docentes pueden grabar sus clases y ponerlas en un repositorio. Así mismo, se puede tener acceso como profesora a las estadísticas de ese espacio, pudiendo identificar cuáles son las secciones más visitadas por los/as alumnos/as.

Las distintas actividades o tareas se diseñaron teniendo en cuenta las indicaciones sobre las cuestiones pragmáticas de la escritura indicadas por Fordyce (2013), Aull (2015) y Liu y Stapleton (2018), así como las consideraciones sobre las actividades propuestas para efectuar la evaluación formativa de Gulikers et al. (2013), Hamodi et al. (2015), Fernando (2018) y Yin y Buck (2019). Se diseñaron un total de diez tareas en cada asignatura. Se tuvo en cuenta que en el grupo de control las tareas se evaluaban de forma sumativa y, por ello, se diseñaron las siguientes pruebas: dos exámenes escritos, cuatro en formato test para 
que fueran contestados por los/as alumnos/as online después de cada unidad, dos proyectos y una memoria. Adicionalmente, se realizó un examen oral en la plataforma Microsoft Teams. Todas las actividades se evaluaron con un rango que variaba desde 0 (mínimo) a 10 (máximo) y la nota final (rango 0-10) resultaba de calcular el peso ponderado de cada actividad y sumarlas para obtener la nota global.

En el grupo experimental se diseñaron diez tareas teniendo en cuenta que se realizaba una evaluación formativa. Las actividades creadas para tal fin fueron: una tarea colaborativa grupal de discusión en el chat sobre uno de los temas aprendidos en clase, dos tareas basadas en estudios de casos, que se tenían que contestar de forma colaborativa con el profesor, tres de ellas que eran un debate por el foro y cuatro tareas que eran exposiciones orales realizadas en la plataforma Microsoft Teams sobre temas tratados en cuatro unidades distintas. Las exposiciones orales se evaluaron proporcionándole retroalimentación a los/as alumnos/as: los/as alumnos/as lo hicieron por el chat y el profesor directamente. La nota final de la asignatura se calculó teniendo en cuenta la participación en las actividades, el producto final de cada tarea, la consecución de las competencias de las asignaturas y la retroalimentación que recibían de los compañeros y la profesora.

Una vez finalizadas las veinte tareas adecuadas a cada tipo de evaluación, se procedió a recopilar las realizadas por los/as alumnos/as después de acabar las clases de las asignaturas del primer y segundo cuatrimestre (las asignaturas se imparten de forma intensiva en un mes) para componer el corpus del grupo de control y el corpus del grupo experimental. A continuación, se recopilaron las distintas evaluaciones y resultados para comparar el progreso de los/as alumnos/as durante el desarrollo de las dos asignaturas. Seguidamente, se estudió el uso de los marcadores textuales e interpersonales en el grupo de control y el experimental para identificar si se detectaban diferencias en los dos corpus analizados con el fin de analizar su capacidad expositiva y persuasiva. Por último, se analizaron los chats de las asignaturas y el foro para identificar la interacción entre profesores y alumnos durante las actividades que se realizaban online, prestando especial atención a los actos comunicativos. 
Los resultados de los análisis se reflejaron en tablas que muestran el rendimiento de los/as alumnos/as, el uso de marcadores metadiscursivos en las tareas, y la interacción realizada en los dos grupos con el profesor y entre los/as alumnos/as. Para finalizar esta investigación, en las conclusiones se comentaron los objetivos conseguidos y se contestaron las preguntas de investigación.

\section{Resultados y discusión}

Los resultados del análisis se han descrito teniendo en cuenta los distintos objetivos que se trazaron en este artículo. Se han comparado en cada sección los resultados del grupo de control y el grupo experimental.

\section{1. Resultados del rendimiento de los/as alumnos/as}

Una vez recopiladas y analizadas las tareas y calculado el rendimiento por tareas, la Tabla 3 muestra los resultados del grupo de control y el experimental:

Tabla 3.

Rendimiento por tareas

\begin{tabular}{lcc}
\hline Tareas & $\begin{array}{c}\text { Nota media del grupo de } \\
\text { control }\end{array}$ & $\begin{array}{c}\text { Nota media del grupo } \\
\text { experimental }\end{array}$ \\
\hline Actividad 1 & 7,6 & 8,0 \\
Actividad 2 & 6,5 & 8,5 \\
Actividad 3 & 6,3 & 8,7 \\
Actividad 4 & 6,4 & 8,8 \\
Actividad 5 & 5,9 & 8,6 \\
Actividad 6 & 6,8 & 8,9 \\
Actividad 7 & 7,3 & 8,0 \\
Actividad 8 & 7,2 & 8,7 \\
Actividad 9 & 7,1 & 8,6 \\
Actividad 10 & 7,5 & 8,9 \\
Nota media total: & 6,86 & 8,57 \\
\hline
\end{tabular}

Fuente: Elaboración propia 
Como se observa en la tabla, el grupo experimental obtuvo una nota media total mayor en la evaluación formativa recibida al realizar las actividades programadas y comentadas entre los compañeros y/o el profesor. Así mismo, si se analizan las distintas tareas, el grupo experimental es el que ha rendido más en la realización de las tareas. Este hecho podría deberse a que, en general, una de las dificultades de los sinohablantes es que no comprendían completamente las implicaciones de las tareas y cuando se realizó la evaluación de forma sumativa (grupo de control) su rendimiento fue menor. Por el contrario, en el grupo experimental, el profesor y los mismos compañeros explicaron las tareas e interactuaron entre sí para lograr un pleno rendimiento. Las dudas se resolvían, se comentaban las posibles respuestas y se ahondaba en los conceptos que no se habían entendido en las explicaciones. Una muestra de este hecho se puede observar en el ejemplo (1):

(1) Alumno: "Ahora tengo una duda. Respecto a la tarea 10, por ejemplo, la primera: La forma de análisis que piensas utilizar en tu Trabajo Fin de Máster (o que te parece más adecuada). Creo que no la entiendo. ¿Cuál es la forma de análisis? ¿Me podrías dar pocos ejemplos? gracias de antemano!" Profesor: "La forma de análisis puede ser de análisis cuantitativo, cualitativo, con corpus de observación, etc".

En la evaluación formativa las alumnas se sintieron respaldadas y se les respondieron sus dudas con el fin de formarles a través de la evaluación. Así mismo, también se observó, en las estadísticas que arroja PoliformaT, que el tiempo medio de estancia por visita de las alumnas del grupo experimental es de 49 minutos, mientras que esta cifra es de 25,8 minutos en los/as alumnos/as del grupo de control. Por lo tanto, esto podría significar que las alumnas que recibieron una evaluación formativa estuvieron más tiempo consultando el material online para realizar las actividades que los/as alumnos/as del grupo de control al que se realizó una evaluación sumativa y esto puede haber influido en sus resultados finales. 


\section{2. Uso de los marcadores metadiscursivos en las tareas}

Una vez recopiladas todas las actividades se identificaron los marcadores textuales e interpersonales utilizados en el grupo de control y el grupo experimental, para comparar las diferencias en el uso y, por lo tanto, su capacidad comunicativa, si lo habían hecho de forma efectiva o no mediante el uso de elementos retóricos. Para extraer estos resultados se recopilaron un total de 70.800 palabras de cada corpus. Se recopiló el mismo número de palabras para que los resultados fueran representativos y no fuera necesario normalizarlos. Los marcadores metadiscursivos identificados se muestran en la Tabla 4 por grupos y tipo de marcador:

\section{Tabla 4}

Rendimiento por tareas

\begin{tabular}{lcc}
\hline Marcadores & Grupo de control & Grupo experimental \\
\hline Textuales & 1.660 & 2.228 \\
- Lógicos & 423 & 514 \\
- Códigos de glosa & 212 & 253 \\
- Secuenciadores & & \\
- Topicalizadores & 289 & 432 \\
- Marcadores del discurso & 248 & 321 \\
- Enunciadores & 183 & 254 \\
- Endofóricos & 98 & 112 \\
- Evidenciales & 125 & 235 \\
& 82 & 107 \\
Interpersonales & & 1.477 \\
- Atenuadores & 1.277 & 320 \\
- Enfatizadores & 288 & 432 \\
- Actitudinales & 354 & 510 \\
- Relacionales & 479 & 113 \\
- Automenciones & 67 & 102 \\
Total & 89 & 3.705 \\
\hline
\end{tabular}

Fuente: Elaboración propia

Se puede observar en la Tabla 4 que tanto el grupo experimental como el de control utilizan más marcadores textuales, hecho ya constatado por otros investigadores en otros corpus (Hyland, 2005), por lo tanto, esto no sería una característica a destacar ya que es un caso recurrente en las lenguas. 
Centrándonos en las diferencias entre los dos grupos de alumnos, se observa que a los que se les realizó una evaluación formativa utilizaron más marcadores discursivos que los que recibieron una evaluación sumativa. Este hecho se puede deber a que reciben información sobre sus tareas, se les comentan sus resultados y se les corrige de forma argumentativa. En el caso del grupo de control, los/as alumnos/as recibieron sus notas por la plataforma online, pero, a pesar de darles la opción de revisar los exámenes, ninguno quiso hacerlo. Ello puede deberse a que no existe una tradición cultural en los/as alumnos/as sinohablantes que les empuje a cuestionar los resultados o a recibir retroalimentación, por ello, este experimento fue un reto tanto desde una perspectiva educativa como cultural.

Analizando los casos de marcadores metadiscursivos, se observa que utilizan los dos grupos un total de 6.642 marcadores en 141.600 palabras. Esto representa que usan, globalmente, 46,9 marcadores por 1.000 palabras. Es un valor bajo, pero tenemos que tener en cuenta que estamos analizando el lenguaje de las actividades que, en muchas ocasiones, es directo y no incluye una gran cantidad de expresiones persuasivas, sino expositivas. Si comparamos los resultados por grupos, se observa que el grupo experimental utiliza más marcadores metadiscursivos, con lo cual se puede decir que sus componentes poseen una mayor capacidad argumentativa y persuasiva que el grupo de control.

\section{3. Interacción con el profesor y entre alumnos}

Para evaluar la interacción y los actos comunicativos entre los/as alumnos/as y entre los/as alumnos/as y los profesores se analizaron los chats y las grabaciones de las presentaciones realizadas en Microsoft Teams y el chat y el foro de PoliformaT. Los resultados se midieron con los siguientes valores adoptando la escala de Likert: habitual: 2; frecuente: 1,5; raro: 1 ; pocas veces: 0,5 ; nunca: 0 . Los resultados más habituales en los dos grupos se muestran en la Tabla 5: 


\section{Tabla 5}

Interacciones

\begin{tabular}{lcc}
\hline Interacciones & Grupo de control & Grupo experimental \\
\hline Chat Teams & raro & habitual \\
Chat PoliformaT & frecuente & habitual \\
Foro & nunca & pocas veces \\
Actividades orales & frecuente & habitual \\
\hline
\end{tabular}

Fuente: Elaboración propia

Los/as alumnos/as interactuaron más con los compañeros o los profesores en el grupo experimental. En el grupo de control, los/as alumnos/as participaban en actos comunicativos si consideraban que podían ser puntuados por la actividad. Sin embargo, las alumnas del grupo experimental utilizan el chat de Teams para interactuar con el profesor, así como el chat de PoliformaT para hacer preguntas sobre un tema o ser graciosos, como se observa en el ejemplo (2):

(2): Alumno: "En este caso, querría preguntarle cómo solucionarlo mejor?" Profesor: "Ya te lo cambié para que pudieras mandármelo, verdad?" Alumno: "Siiii, lo he visto el cambio, muchas gracias (-), jajaja."

Como se puede observar, los actos comunicativos son distendidos, sin formalismos, con el profesor. Este ejemplo es del grupo experimental, en el que se observa que no hay tensión y que las alumnas están acostumbradas a hablar con la profesora puesto que lo hacen frecuentemente en la evaluación formativa. La Figura 3 nos muestra un chat y como se desarrolla online en PoliformaT: 


\section{Figura 3}

Chat de la asignatura en PoliformaT anonimizado

Fuente: PoliformaT

A continuación, en la Figura 4 se muestra un chat en Teams, en el que se preguntan cuestiones académicas sobre la asignatura. El tono es cordial y distendido, con lo cual se ha logrado la interacción entre alumno y profesor. La interacción entre alumnos se pudo observar más en las exposiciones orales y en el chat de PoliformaT. Ello no indica que los/as alumnos/as chinos/as se comuniquen de forma fluida y constante, ya que se observó durante el desarrollo de las clases que para ellos/as la comunicación en español es complicada y prefieren el silencio a la participación. Este silencio se observó que era más acentuado en las clases online síncronas. Por ello, la profesora tenía que utilizar diversas estrategias para propiciar la interacción entre los/as alumnos/as o entre alumnos/as y profesora. 


\section{Figura 4}

\section{Chat de Microsoft Teams anonimizado}

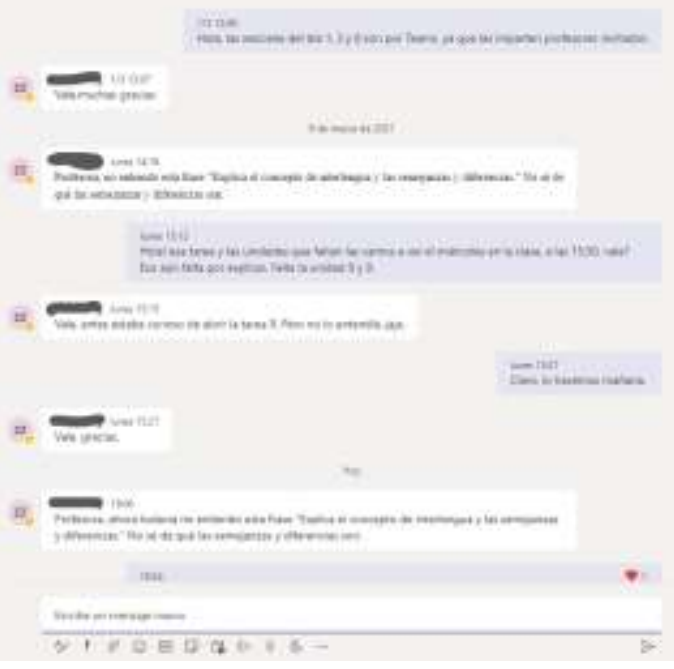

Fuente: Microsoft Teams

Finalmente, después de mostrar los resultados según los objetivos planteados, vamos a pasar a las conclusiones del estudio.

\section{Conclusiones}

Este estudio nos ha permitido comparar dos formas de evaluación que se ha llevado a cabo con un grupo de estudiantes sinohablantes para poder comprobar si existen diferencias en los resultados obtenidos por los/as alumnos/as a nivel de rendimiento, retórico y comunicativo. El primer objetivo que tracé era identificar el rendimiento por parte de los alumnos sinohablantes. Hemos podido comprobar en los resultados que sí que se ha detectado un mayor progreso en los resultados de la evaluación formativa, que puede estar inducido por un uso más efectivo de la lengua española al tener la posibilidad de comentar con la profesora y alumnas sus dudas sobre las actividades con más naturalidad y no sujetos a una revisión de las tareas una vez evaluadas.

El segundo objetivo era identificar si el uso de los marcadores metadiscursivos $\mathrm{y}$, con ello, la capacidad expositiva y persuasiva de 
los/as alumnos/as, mejoraba con la evaluación formativa. A través del análisis del uso de los marcadores se ha podido afirmar que el grupo experimental ha utilizado más estrategias retóricas, pero tengo que ser cauta ante esta afirmación ya que se trata de un uso escaso si tenemos en cuenta el total de palabras de los dos corpus. La interacción que se favorece en la evaluación formativa consideramos que es beneficiosa para mejorar la comunicación, como también indican Schildkamp et al. (2020) y Granberg et al. (2021). También considero que el uso de plataformas para la evaluación formativa ha sido beneficioso, ya que los alumnos han participado más en el grupo experimental que en el grupo de control.

Respecto al tercer objetivo, se analizaron las interacciones entre profesores y alumnos en las actividades online, y se comprobó que los/as alumnos/as preferían la interacción en las actividades orales por Teams y por el chat de PoliformaT. Estas interacciones fueron en un $80 \%$ guiadas por el profesor, ya que se comprobó que la interacción espontánea era difícil de conseguir en la asignatura online en los dos grupos.

En resumen, puedo afirmar que la evaluación formativa es más efectiva que la evaluación sumativa para incentivar la comunicación entre alumnos y mejorar la producción de la lengua meta. Este hecho ya ha sido también notado por autores como Gulikers et al (2013) y Yin y Buck (2019). He observado que la interacción entre profesor y alumno en la evaluación formativa es más efectiva que en la evaluación sumativa en las actividades online, aunque como indican Pachler et al. (2010, p. 720) "[...] no technology-based assessment is in itself formative, but almost any technology can be used in a formative way". Soy consciente de que este estudio ha de ampliarse con más muestras para poder extrapolar los resultados; la razón de en este análisis es aportar valores que pueden ser utilizados en otros estudios para medir la efectividad de distintos tipos de evaluación. 


\section{Referencias bibliográficas}

Albelda, M., y Cestero, A. (2011). De nuevo sobre los procedimientos de atenuación. Español Actual, 96, 121-155.

Andersson, C., y Palm, T. (2017). The impact of formative assessment on student achievement: A study of the effects of changes to classroom practice after a comprehensive professional development programme. Learning and Instruction, 49, 92-102. doi: 10.1016/j.learninstruc.2016.12.006.

Aull, L. (2015). Connecting writing and language in assessment: Examining style, tone, and argument in the U.S. Common Core standards and in exemplary student writing. Assessing Writing, 24, 59-73. doi: 10.1016/j.asw.2015.03.002.

Black, P. (2015). Formative assessment - an optimistic but incomplete vision, Assessment in Education: Principles, Policy \& Practice, 22(1), 161-177. doi: 10.1080/0969594X.2014.999643.

Black, P. (2010). Formative Assessment. En P. Peterson, E. Baker, y B. McGaw (Eds.), International Encyclopedia of Education (pp. 359-364). Elsevier.

Briz, A. (2007). Para un análisis semántico, pragmático y sociopragmático de la cortesía atenuadora en España y América. Lingüística Española Actual, 29(1), 5-40.

Carrió-Pastor, M. L. (2016). A contrastive study of the hedges used by English, Spanish and Chinese researchers in academic papers. En F. Alonso Almeida et al. (Eds.), Input a word, analyze the world: Selected approaches to Corpus Linguistics (pp. 477-492). Cambridge Scholars.

Carrió-Pastor, M. L. (2019). Different ways to express personal attitudes in Spanish and English engineering papers: An analysis of metadiscourse devices, affective evaluation and sentiment analysis. Lodz Papers in Pragmatics, 15(1), 45-67. doi: 10.1515/lpp2019-0004.

Carrió-Pastor, M. L. (2020). Conocer la lengua a través de los corpus: la herramienta METOOL, retos para el análisis de los marcadores discursivos. Pragmalingüística, 28, 255-274. doi: 10.25267/Pragmalinguistica.2020.i28.13. 
Carrió-Pastor, M. L. (2021, en prensa). Emphasising and mitigating statements in linguistics and engineering academic papers written by non-native speakers of English. En I. Kecskes (Ed.), Cambridge Handbook of Intercultural Pragmatics (pp. en prensa). Cambridge University Press.

Connor, U. (1996). Contrastive Rhetoric: Cross-Cultural Aspects of Second Language Writing. Cambridge University Press.

Costa, D. S. J., Mullan, B. A., Kothe, E. J., y Butow, P. (2010). A web-based assessment tool for Master students: A pilot study. Computers \& Education, 54, 1248-1253. doi: 10.1016/j.compedu.2009.11.011.

Crystal, D. (1997). A dictionary of linguistics and phonetics. Blackwell.

De la Hoz Ibacache, S. (2016). Objetivos y metodologías en los estudios de evaluación formativa de la composición escrita entre el periodo de 1992 y 2014. Tejuelo, 24, 31-36. doi: 10.17398/19888430.24.1.31.

Fernando, W. (2018). Show me your true colours: Scaffolding formative academic literacy assessment through an online learning platform. Assessing Writing, 36, 63-76. doi: 10.1016/j.asw.2018.03.005.

Fernando, W. (2020). Moodle quizzes and their usability for formative assessment of academic writing. Assessing Writing, 46, 1-6. doi: 10.1016/j.asw.2020.100485.

Flores, N. (2020). Linguistic mitigation in English and Spanish: How speakers attenuate expressions. Routledge.

Fordyce, K. (2013). The differential effects of explicit and implicit instruction on EFL learners' use of epistemic stance. Applied Linguistics, 35, 6-28. doi: 10.1093/applin/ams076.

Gikandi, J. W., Morrow, D., y Davis, N. E. (2011). Online formative assessment in higher education: A review of the literature. Computers \& Education, 57, 2333-2351. doi: 10.1016/j.compedu.2011.06.004.

Granberg, C., Palm, T., y Palmberg, B. (2021). A case study of a formative assessment practice and the effects on students' selfregulated learning. Studies in Educational Evaluation, 68, 1-10. doi: 10.1016/j.stueduc.2020.100955. 
Gulikers, J. T. M., Biemans, H. J. A., Wesselink, R., y van der Wel, M. (2013). Aligning formative and summative assessments: a collaborative action research challenging teacher conceptions. Studies in Educational Evaluation, 39, 116-124. doi: 10.1016/j.stueduc.2013.03.001.

Halliday, M. A. K. (1998). El lenguaje como semiótica social. La interpretación social del lenguaje y su significado. S. L. Fondo de cultura económica de España.

Hamodi, C., López Pastor, V. M., y López Pastor, A. T. (2015). Medios, técnicas e instrumentos de evaluación formativa y compartida del aprendizaje en educación superior. Perfiles educativos, 147, 146-161.

Hansen, G., y Ringdal, R. (2018). Formative assessment as a future step in maintaining the mastery-approach and performanceavoidance goal stability. Studies in Educational Evaluation, 56, 59-70. doi: 10.1016/j.stueduc.2017.11.005.

Hansen, G. (2020). Formative assessment as a collaborative act. Teachers' intention and students' experience: Two sides of the same coin, or? Studies in Educational Evaluation, 66, 1-10. doi: 10.1016/j.stueduc.2020.100904.

Havnes, A., Smith, K., Dyste, O., y Ludvigsen, K. (2012). Formative assessment and feedback: Making learning visible. Studies in Educational Evaluation, 38, 21-27. doi: 10.1016/j.stueduc.2012.04.001.

Hyland, K. (1998). Persuasion and context: The pragmatics of academic metadiscourse. Journal of Pragmatics, 30, 437-455.

Hyland, K. (2005). Metadiscourse: Exploring Interaction in Writing. Continuum.

Liu, F., y Stapleton, P. (2018). Connecting writing assessment with critical thinking: An exploratory study of alternative rhetorical functions and objects of enquiry in writing prompts. Assessing Writing, 38, 10-20. doi: 10.1016/j.asw.2018.09.001.

Marshall, B. (2010). Formative Assessment and Instructional Planning. En P. Peterson, E. Baker, y B. McGaw (Eds.), International Encyclopedia of Education (pp. 365-368). Elsevier.

Meusen-Beekman, K. D., Brinke, D. J., y Boshuizen, H. P. A. (2016). Effects of formative assessments to develop self-regulation among sixth grade students: Results from a randomized controlled 
intervention. Studies in Educational Evaluation, 51, 126-136. doi: 10.1016/j.stueduc.2016.10.008.

Mohamadi, Z. (2018). Comparative effect of online summative and formative assessment on EFL student writing ability. Studies in Educational Evaluation, 59, 29-40. doi: 10.1016/j.stueduc.2018.02.003.

Moya, P., y Carrió-Pastor, M. L. (2018). Estrategias de intensificación en los comentarios digitales sobre noticias. Spanish in Context, 15 (3), 369-391. doi: 10.1075/sic.00019.car.

Mur Dueñas, P. (2011). An intercultural analysis of metadiscourse features in research articles written in English and in Spanish. Journal of Pragmatics, 43, 3068-3079. doi: 10.1016/j.pragma.2011.05.002.

Panadero, E., y Jonsson, A. (2013). The use of scoring rubrics for formative assessment purposes revisited: A review. Educational Research Review, 9, 129-144. doi: 10.1016/j.edurev.2013.01.002.

Pachler, N., Daly, C., Mor, Y., y Mellar, H. (2010). Formative e-assessment: Practitioner cases. Computers \& Education, 54, 715-721. doi: 10.1016/j.compedu.2009.09.032.

Pellegrino, J. W. (2010). Technology and formative assessment. En P. Peterson, E. Baker, y B. McGaw (Eds.), International Encyclopedia of Education (pp. 43-47). Elsevier.

Pine, G. J. (2009). Teacher action research: Building knowledge democracies. Sage.

Pla-Campas, G., Arumí-Prat, J., Senye-Mir, A. M., y Ramírez, E. (2016). Effect of using formative assessment techniques on students' grades. Procedia. Social and Behavioural Sciences, 228, 190-195. doi: 10.1016/j.sbspro.2016.07.028.

Roever, C. (2014). Assessing Pragmatics. En A. J. Kunnan (Ed.), Companion to Language Assessment (pp. 1-15). Wiley.

Salas Valdebenito, M. (2015). Una propuesta de taxonomía de marcadores metadiscursivos para el discurso académico-científico escrito en español. Revista Signos, 48 (87), 95-120. doi: 10.4067/S0718-09342015000100005.

Schildkamp, K., Van der Kleij, F. M., Heitink, M. C., Kippers, W. B., y Veldkamp, B. P. (2020). Formative assessment: A systematic review of critical teacher prerequisites for classroom practice. 
International Journal of Educational Research, 103, 1-16. doi: 10.1016/j.ijer.2020.101602.

Sutton, R. (2010). Challenges of developing and implementing formative assessment practices in schools. En P. Peterson, E. Baker, y Barry McGaw (Eds.), International Encyclopedia of Education (Third Edition), (pp. 353-358). Elsevier.

Talanquer, V. (2015). La importancia de la evaluación formativa. Educación Química, 26, 177-179. doi: 10.1016/j.eq.2015.05.001

Thompson, G. (2001). Interaction in Academic Writing: Learning to Argue with the Reader. Applied Linguistics 22(1), 58-78. doi: 10.1093/applin/22.1.58.

Tillema, H. (2010). Formative Assessment in Teacher Education and Teacher Professional Development. En P. Peterson, E. Baker, y B. McGaw (Eds.), International Encyclopedia of Education (Third Edition) (pp. 563-571). Elsevier.

Van der Kleij, F. M. (2019). Comparison of teacher and student perceptions of formative assessment feedback practices and association with individual student characteristics. Teaching and Teacher Education, 85, 175-189. doi: 10.1016/j.tate.2019.06.010

Wilson, J., Roscoe, R., y Ahmed, Y. (2017). Automated formative writing assessment using a levels of language framework. Assessing Writing, 34, 16-36. doi: 10.1016/j.asw.2017.08.002.

Xiao, Y., y Yang, M. (2019). Formative assessment and selfregulated learning: How formative assessment supports students' selfregulation in English language learning. System, 81, 39-49. doi: 10.1016/j.system.2019.01.004.

Yin, X., y Buck, G. A. (2019). Using a collaborative action research approach to negotiate an understanding of formative assessment in an era of accountability testing. Teaching and Teacher Education, 80, 27-38. doi: 10.1016/j.tate.2018.12.018. 
Tejuelo, $\mathrm{n}^{\circ} 34$ (2021), págs. 261-292. Análisis comparativo del uso de marcadores metadiscursivos... 